\title{
Mycobacterium chelonei keratitis: a case report
}

\author{
DONALD J. MIRATE,' DAVID S. HULL, ' JOHN H. STEEL, JR., ${ }^{2}$ AND \\ MARY JO CARTER ${ }^{3}$ \\ From the Departments of 'Ophthalmology, ${ }^{2}$ Pathology, and ${ }^{3}$ Medicine, Medical College of Georgia, Augusta, \\ Georgia 30912, USA
}

SUMmARY The case is reported of a patient who initially presented with a dendritiform corneal ulcer that ultimately failed to heal and in which Mycobacterium chelonei was repeatedly cultured. The organism was sensitive only to kanamycin and amikacin; however, topical administration of these antibiotics failed to achieve a complete cure. Penetrating keratoplasty was ultimately required to eradicate the organism from the cornea.

Mycobacterium chelonei, along with Mycobacterium fortuitum, belongs to group IV (rapid growers) in Runyon's classification of atypical mycobacteria.' They are differentiated in the laboratory by the nitrate reduction test and iron uptake test: both tests are negative for Mycobacterium chelonei and positive for Mycobacterium fortuitum. ${ }^{2}$ Treatment is difficult and protracted because both pathogens are resistant to most antituberculous drugs and are generally sensitive only to kanamycin or amikacin. ${ }^{3-5} \mathrm{Myco}$. fortuitum is probably a more common cause of keratitis, with at least 9 cases having been reported ${ }^{6-10} \mathrm{We}$ are aware of only 2 cases of keratitis due to the Myco. chelonei. ${ }^{511}$ In this paper we describe the course of a patient with an indolent corneal ulcer from which Mycobacterium chelonei was isolated and who ultimately required penetrating keratoplasty.

\section{Case report}

An otherwise healthy 84-year-old white woman complained of a foreign body sensation in the left eye after raking leaves. Her ophthalmologist noted a dentritic-like ulcer, and treated it for 5 weeks with idoxuridine, trifluoridine, and a combination of sulphacetamide, prednisoline acetate, and phenylephrine. After an initial improvement there was no further healing, so she was referred to the Department of Ophthalmology, Medical College of Georgia.

Her visual acuity was 20/50 OD (aphakic with hard contact lens) and finger counting at 1 foot $(30 \mathrm{~cm}) \mathrm{OS}$.

Correspondence to David S. Hull, MD. Department of Ophthalmology, Medical College of Georgia. Augusta, GA 30912, USA.
There was a mild entropion of the left lower lid and a moderate ciliary flush. A linear $1 \mathrm{~mm}$ central epithelial defect was surrounded by a faint subepithelial infiltrate with scarring and discrete punctate epithelial erosions. The anterior chamber was deep and clear.

She was treated for a clinically apparent postinfectious ulcer with erythromycin ophthalmic ointment and pressure patching; however, the defect did not heal. After 18 days' therapy the cornea was scraped for culture and plated on blood agar. and the medication was changed to a combination polymyxin B, neomycin, and gramicidin ophthalmic drops every 2 hours. A Fox procedure was performed to correct the entropion. By the fifth day a confluent growth of acid-fast bacilli was present on the blood agar which was subsequently identified Mycobacterium chelonei.

A 5\% hypopyon developed, and the patient was admitted to the Talmadge Memorial Hospital for more intensive treatment. The initial therapy suggested by the infectious diseases consultant was topical kanamycin $30 \mathrm{mg} / \mathrm{ml}$ drops hourly, alternating at 30-minute intervals with topical doxycyline 30 $\mathrm{mg} / \mathrm{ml}$ drops hourly. The cornea was again scraped for culture prior to starting these antibiotics and again yielded Myco. chelonei. Because of the lack of response the previous medications were stopped, and topical amikacin $30 \mathrm{mg} / \mathrm{ml}$ drops every half hour were begun. During the next few days the epithelial defect decreased in size, the infiltrate showed evidence of clearing. and the hypopyon resolved. She was discharged from the hospital with erythromycin ophthalmic ointment as the only medication. One month later the epithelium had healed, but the visual acuity was unchanged.

The ciliary flush then increased after 3 weeks and 


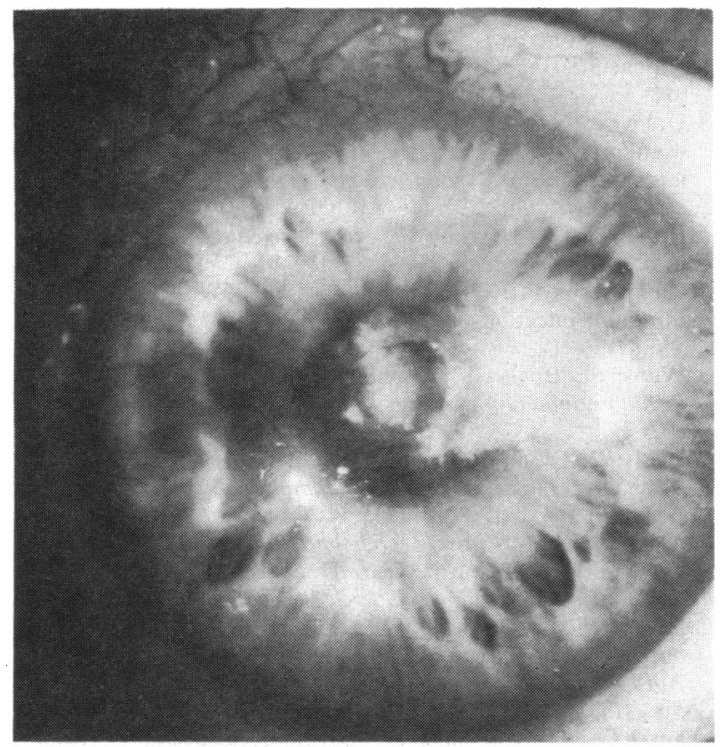

Fig. 1 Appearance of mycobacterium chelonei corneal ulcer at the time of recurrence.

was accompanied by an anterior chamber reaction. A 'cracked windshield' appearance of the cornea was noted; however, the corneal epithelium was intact. Prednisolone sodium phosphate $\mathbf{0} \cdot 125 \%$ twice daily was reluctantly added. The anterior chamber reaction and the patient's discomfort increased further during the next few days, and the prednisolone sodium phosphate was increased to $1 \% 4$ times a day. The ulcer recurred in 4 weeks (Fig. 1), and she was treated in the hospital with topical amikacin $30 \mathrm{mg} / \mathrm{ml}$ drops and sulphacetamide $30 \%$ hourly. The ulcer did not resolve, and repeat cultures continued to yield acid-fast organisms. Because of the lack of microbiological and clinical response a $7.5 \mathrm{~mm}$ penetrating keratoplasty was performed on 24 March 1982. Initial postoperative medications were topical amikacin 30

Table 1 Antimicrobial agent sensitivity of Mycobacterium chelonei isolated in this case

\begin{tabular}{lc}
\hline Agent & MIC $\mu \mathrm{g} / \mathrm{ml}$ \\
\hline Amikacin & 4 \\
Cefotaxime & 64 \\
Cefoxitin & 32 \\
Doxycycline & $>16$ \\
Erythromycin & $>16$ \\
Gentamicin & 16 \\
Kanamycin & 4 \\
Moxalactam & $>128$ \\
Sulphamethoxazole & 256 \\
Ethionamide & 128 \\
Tobramycin & 8 \\
\hline
\end{tabular}

MIC = minimal inhibitory concentration in broth. $\mathrm{mg} / \mathrm{ml}$ drops 4 times a day and prednisone $20 \mathrm{mg}$ orally per day. Six months later the graft was clear and the patient was comfortable, and there was no evidence of recurrence. Mycobacterium chelonei sensitive only to amikacin and kanamycin (Table 1) was isolated from 7 separate cultures, including that of the excised cornea, taken over a 6-month period.

\section{Discussion}

We believe this is the third published case of Mycobacterium chelonei keratitis. The initial presentation of a dendritic ulcer and infiltrate with a history of antiviral medication was similar to the case reported by Gangadharam et al. ${ }^{11}$ A 'cracked windshield' appearance of the cornea, which was described by Lazar et al. ${ }^{10}$ as an early, transient, and almost pathognomonic sign of Myco. fortuitum keratitis, did not occur until late in this case and persisted for several weeks.

Sensitivity of the organism to amikacin and kanamycin was consistent with reports of in-vitro disc diffusion and agar dilution tests, ${ }^{34}$ and with drug sensitivities in reports of Myco. chelonei infections other than keratitis. ${ }^{12}{ }^{13}$ A previous report had noted that amikacin inhibited $100 \%$ of Myco. fortuitum isolates at $2 \mu \mathrm{g} / \mathrm{ml}$ whereas $91 \%$ of Myco. chelonei had minimum inhibitory concentrations of $4 \mu \mathrm{g} / \mathrm{ml}$ or greater, ${ }^{4}$ as was found in this case (Table 1 ). We are not certain what levels of amikacin were achieved in the corneal stroma during treatment. It has also been previously reported that doxycycline is useful in the treatment of this organism 5 and that $44 \%$ of strains are inhibited by $4 \mu \mathrm{g} / \mathrm{ml}$ doxycycline with $14 \%$ of strains being susceptible to $2 \mu \mathrm{g} / \mathrm{ml}$ erythromycin. ${ }^{4}$ The minimum inhibitory concentration for this organism was greater than $16 \mu \mathrm{g} / \mathrm{ml}$ for both doxycycline and erythromycin (Table 1). A previously reported case of Myco. chelonei keratitis was sensitive only to gentamicin ${ }^{11}$; however, resistance to amikicin or kanamycin was not specifically noted. This organism was sensitive to $16 \mu \mathrm{g} / \mathrm{ml}$ gentamicin.

We are confident that the organism was indeed Mycobacterium chelonei, since it was isolated from multiple cultures taken weeks apart and characterised by 2 separate laboratories. Acid-fast bacilli and granulomatous inflammation were not found in the recipient corneal specimen, which showed only nonspecific inflammation. This was consistent with the repeated difficulty in finding histological evidence of mycobacteria in experimentally produced rabbit Mycobacterium fortuitum infections as reported by Kirber et al. ${ }^{14}$ While we were initially successful in healing the ulcer and promoting re-epithelialisation by a prolonged course of topical medications, the organism was evidently still present in the corneal 
stroma. The necessity of treating her iritis with topical steroids after epithelialisation was accomplished may have hastened the emergence of recurrent disease, which ultimately required a penetrating keratoplasty for definitive treatment.

The treatment of infections of the soft tissues with Myco. fortuitum or Myco. chelonei has frequently included excision or debridement. ${ }^{5}$ Of the two previously reported cases of $M y c o$. chelonei keratitis one was successfully treated with topical amikacin, ${ }^{5}$ but the other required penetrating keratoplasty. ${ }^{.1}$ This case has demonstrated the potential value of penetrating keratoplasty, if it is done while adequate infection-free margins are still present, for the treatment of Myco. chelonei keratitis that fails to respond to medical therapy.

\section{References}

1 Ward JM. $M$. fortuitum and $M$. chelonei-fast growing mycobacteria. Br J Dermatol 1975; 92: 453-9.

2 Vestal AL. Procedures for the isolation and identification of mycobacteria. HEW Publication (CDC) 1975; 79-8230: 54.

3 Dalovisio JR, Pankey GA. In vitro susceptibility of Mycobacterium fortuitum and Mycobacterium chelonei to amikacin. $J$ Infect Dis 1978; 137: 318-21.
4 Wallace RJ Jr, Dalovisio JR. Pankey GA. Disk diffusion testing of Mycobacterium fortuitum and Mycobacterium chelonei to antibacterial agents. Antimicrob Agents Chemother 1979; 16: 611-4.

5 Dalovisio JR, Pankey GA, Wallace RJ. Jones DB. Clinical usefulness of amikacin and doxycycline in the treatment of infection due to Mycobacterium fortuitum and Mycobacterium chelonei. Rev Infect Dis 1981; 3: 1068-74.

6 Levenson DS. Harrison CH. Mycobacterium fortuitum corneal ulcer. Arch Ophthalmol 1966; 75: 189-91.

7 Zimmerman LE. Turner L. McTigue JW. Mycobacterium fortuitum infection of the cornea. Arch Ophthalmol 1969; 82: 596-601.

8 Wunsh SE. Boyle GL. Leopold IH, Littman ML. Mycobacterium fortuitum infection of corneal graft. Arch Ophthalmol 1969; 82: 602-7.

9 Willis WE, Laibson PR. Intractable Mycobacterium fortuitum corneal ulcer in man. Am J Ophthalmol 1971; 71: 500-4.

10 Lazar M. Nemet. P. Bracha R. Campus A. Mycobacterium fortuitum keratitis. Am J Ophthalmol 1974; 78: 530-2.

11 Gangadharam PRJ Jr, Lanier JD, Jones DB. Keratitis due to Mycobacterium chelonei. Tubercle 1978; 59: 55-60.

12 Smith RE, Salz JJ, Moors R, Silverstein D, Lewis W. Mycobacterium chelonei and orbital granuloma after tear duct probing. Am J Ophthalmol 1980; 98: 139-41.

13 Speert DP, Munson D. Mitchell C. Johnson DE. Thompson TR. Ferrieri P. Wannamaker LW. Mycobacterium chelonei septicemia in a premature infant. J Pediatr 1980; 96: 681-3.

14 Kirber MW. Kirber HP. Dubin IN. Experimental intraocular infection with Mycobacterium fortuitum. Am J Ophthalmol 1974: 77: 173-7. 